\title{
A Dual-Band Wearable Exposure Meter for Personal RF Exposure Assessment in Indoor Environments: On-body Calibration in a Reverberation Chamber
}

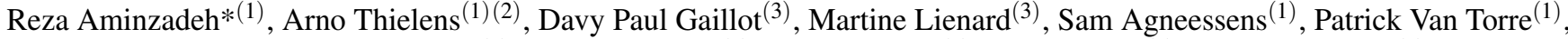 \\ Matthias Van den Bossche ${ }^{(1)}$, Hendrik Rogier $^{(1)}$, Martin Röösli(4) ${ }^{(4)}$ Luc Martens ${ }^{(1)}$ and Wout Joseph ${ }^{(1)}$ \\ (1) Department of Information Technology (INTEC), Ghent University/imec, \\ Technologiepark-Zwijnaarde 15, B 9052 Ghent, Belgium \\ *Correspondence: reza.aminzadeh@ugent.be \\ (2) Berkeley Wireless Research Center, Department of Electrical Engineering and Computer Sciences, \\ University of California, Berkeley, CA 94704, United States \\ (3) Group TELICE, IEMN, University of Lille, Lille, France \\ (4) Swiss Tropical and Public Health Institute/University of Basel, Basel, Switzerland
}

\begin{abstract}
For the first time, a dual band wearable personal distributed exposure meter (DPDE) is designed. The DPDE is calibrated on-body in a reverberation chamber. The meter consists of four nodes including textile antennas operating at $2443 \mathrm{MHz}$ (WiFi $2 \mathrm{GHz}$ ) and $2595 \mathrm{MHz}$ (LTE $2600 \mathrm{MHz}$ ). The location of the nodes are optimized based on the calibration in a reverberation chamber. The results show that the DPDE has a high antenna-body isolation and thus its performance is less affected by presence of the human body. The measurement uncertainty of the designed DPDE is maximum $4.5 \mathrm{~dB}$ in terms of $68 \%$ confidence interval of its response. The results are validated in a real office environment for WiFi $2 \mathrm{GHz}$. The DPDE has a detection limit of $3 \mu \mathrm{W} . \mathrm{m}^{-2}$ in diffuse fields.
\end{abstract}

\section{Introduction}

People spend more than $80 \%$ of their time indoors [1]. Meanwhile, the number wireless devices e.g. tablets and smart phones, is rapidly increasing. This could result in an increasing exposure of people to radio-frequency (RF) electromagnetic fields (EMF). This increase is associated with a growing concern regarding the potential health effects of wireless devices. For instance, the WHO identified characterization of exposure to emerging RF EMF-based technologies as an important issue to be addressed by many countries [2]. The total RF power in an indoor environment is composed of specular and diffuse or dense multipath components (DMC) [3]. This is a result of coherent (large surfaces) and non-coherent (due to the presence of different objects) reflections in an indoor area. The contribution of DMC to the power density in an indoor environment maybe up to $95 \%$ [4]. Therefore, assessment of human exposure to RF sources in indoor environments is crucial.

Personal exposure to RF EMF is measured by personal ex- posure meters (PEMs). Several studies have shown that PEMs are faced with large measurement uncertainties up to $30 \mathrm{~dB}$ [5]. A review of factors contributing to uncertainty of PEMs can be found in [6]. Personal distributed exposure meters (PDEs), including single band [7] or multi-band nodes [8] have been proposed and calibrated on body in anechoic environments. PDEs have an smaller uncertainty compared to PEMs in specular conditions $[7,8]$. However, it is unsure whether this is the case in diffuse RF exposure conditions (i.e. indoor environments). The response of two PEMs under diffuse exposure has been studied using numerical simulations and measurements [9].

In this paper, for the first time, we present the design and on-body calibration of a Dual-band PDE (DPDE) in a reverberation chamber (RC). The DPDE consists of four nodes on the front and back of the user's body. The DPDE measures the incident power density $\left(S_{\text {inc }}\right)$ for the downlink band of Long-Term Evolution (LTE) $2600 \mathrm{MHz}$ and Wireless Fidelity $2 \mathrm{GHz}$ (WiFi-2G).

The methodology is described in Section 2. Section 3 presents the results and Section 4 concludes the paper.

\section{Methodology}

The DPDE consists of 4 nodes (antennas) operating at $2443 \mathrm{MHz}$ and $2595 \mathrm{MHz}$ that cover WiFi-2G and LTE 2600, respectively. The nodes have been calibrated on a 28-year old male subject with a body mass index (BMI) of $23.6 \mathrm{~kg} / \mathrm{m}^{2}$ in an RC. The on-body location of the nodes is predefined and has been studied in [8] (see Figure 1).

\subsection{Nodes}

Figure 2 shows an example of a node which consists of an elliptically polarized Substrate Integrated Waveguide (SIW) textile antenna and a printed circuit board (PCB). 


\begin{tabular}{|c|c|}
\hline A & B \\
\hline C & D \\
\hline E & F \\
\hline G & H \\
\hline I & J \\
\hline \multicolumn{2}{|c|}{ Front }
\end{tabular}

\begin{tabular}{|c|c|}
\hline K & L \\
\hline $\mathbf{M}$ & $\mathbf{N}$ \\
\hline $\mathbf{O}$ & $\mathbf{P}$ \\
\hline $\mathbf{Q}$ & $\mathbf{R}$ \\
\hline $\mathbf{S}$ & $\mathbf{T}$ \\
\hline \multicolumn{2}{|c|}{ Back }
\end{tabular}

Figure 1. The potential locations on body to place the nodes of the DPDE. The optimized locations are: JN (WiFi2G); BT (LTE 2600).

The design of the antennas is based on [10]. Each node has a sampling rate of $1 \mathrm{~Hz}$ and a dynamic range of $80 \mathrm{~dB}$.

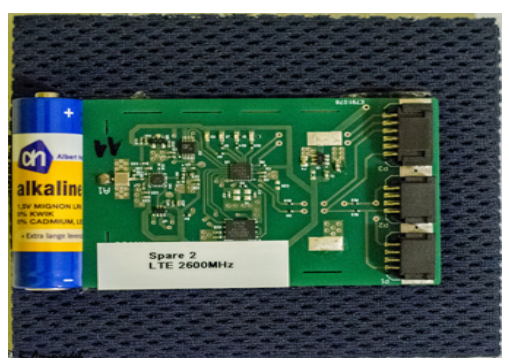

Figure 2. An example of an LTE 2600 node including a textile antenna and a PCB.

\section{2 on-body calibration in the $\mathrm{RC}$}

Figure 3 illustrates the measurement setup in the RC. The room has a volume of $65 \mathrm{~m}^{3}$ and has a stirrer to stir electromagnetic modes.

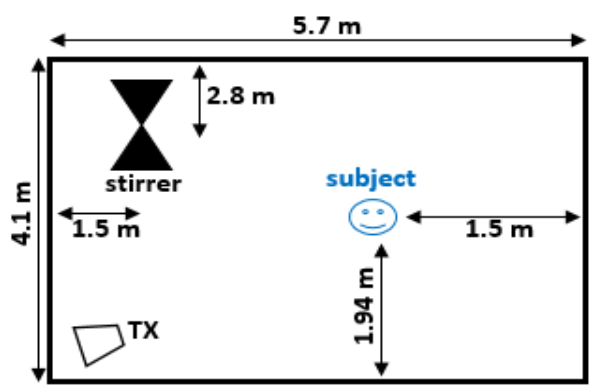

Figure 3. The measurement setup used for on-body calibration in the RC.

First, the subject is placed in the working area of the chamber in the room (see Fig 3). The TX delivers a constant power of $0 \mathrm{dBm}$ and the received power $P_{r}$ is measured on each node on their optimized locations ( $\mathrm{JN}$ for $\mathrm{WiFi}-2 \mathrm{G}$; BT for LTE 2600) during a full rotation of the stirrer (at a speed of $2^{\circ} / s$ ). Second, to study the effect of node's location on the DPDE's measurement uncertainty, the nodes location of the nodes are changed as follows: for WiFi-2G, the node on the front $(\mathrm{J})$ moved from the left side of the trunk to the right side $(\mathrm{G})$, and the node on the back $(\mathrm{N})$ moved from the right to the left of the trunk (M); for LTE
2600, the node on the front (B) moved from down vertically on the left chest (D), and the node on the back (T) moved from the bottom to upper part on the right side of the trunk (L). Third, the first step is repeated for the nodes on their new locations. Fourth, $S_{\text {inc }}$ is measured using a broad band isotropic field meter (Narda NBM 550, Hauppauge, NY) in the empty chamber (at the same location of the subject) at different heights from the floor $(68-201 \mathrm{~cm})$. These values are averaged over the measured heights and the positions of the stirrer. Finally, the antenna aperture (AA) in diffuse fields is obtained for each frequency band from the measurements:

$$
A A=\frac{P_{r}}{S_{i n c}}
$$

where $P_{r}$ is the on-body received power averaged over all positions of the stirrer. The $P_{r}$ for the front and back are then averaged geometrically for each frequency band.

\subsection{Validation in a real indoor environment}

In order to validate the designed DPDE, the incident power densities $S_{\text {inc }}$ are measured using a tri-axial antenna and a spectrum analyzer (R\&S FSL, Rhode \& Schwartz, Munich, Germany). This procedure is proposed in [11]. WiFi-2G is chosen since this is a dominant signal in indoor environments. Two locations are chosen in the 5th floor of an office building in Ghent, Belgium (see Fig. 4). The antenna is placed $1.5 \mathrm{~m}$ above the ground floor on these two locations and $S_{i n c}$ is measured. Following the measurements with the antenna the subject wearing the DPDE walks around a square of $1 \times 1 \mathrm{~m}^{2}$ centered on the two chosen locations. The received power $P_{r}$ is registered on each node during the measurements. Using equation (1), the actual exposure is calculated and is compared to the measured values by the tri-axial antenna.

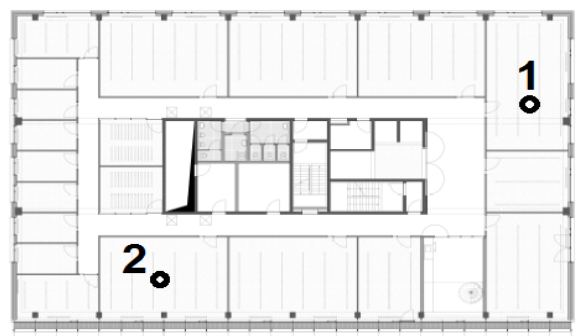

Figure 4. Ground plan of office building used for measurements. The circles show the location of the measurements.

\section{Results and Discussion}

\subsection{On-body calibration in the RC}

Figure 5 demonstrates the $68 \%$ confidence interval $\left(\mathrm{CI}_{68}\right)$ of the on-body AA for WiFi-2G and LTE 2600 in diffuse fields. For both frequency bands and the measured positions of the nodes, the nodes on the front have a $C_{68}$ in the range of 5-6 dB while the nodes on the back have a $C_{68}$ of $6-7 \mathrm{~dB}$. Averaging over the two nodes on the front and back reduces 
the uncertainty to 4 and $4.5 \mathrm{~dB}$ for WiFi-2G (JN) and LTE 2600 (BT), respectively. A $C I_{95}$ of $6.9 \mathrm{~dB}$ for the average response over two PEMs in an RC has been reported in [9].
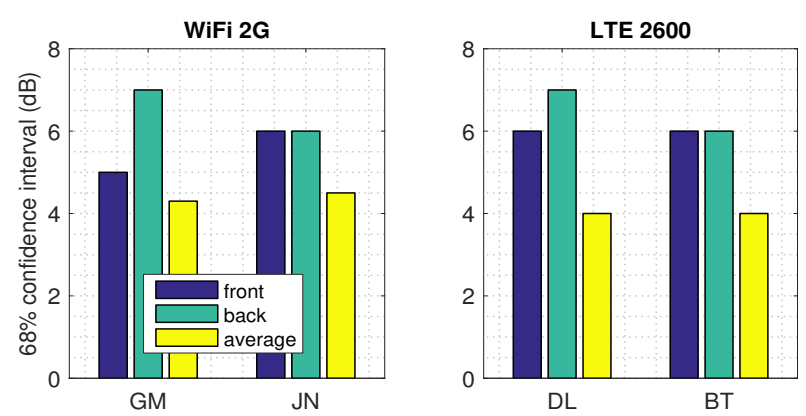

Figure 5. The $68 \%$ confidence interval of the on-body antenna aperture for different locations of the nodes.

Changing the location of the nodes for both frequency bands does not have a significant influence on the uncertainty of the DPDE. For example, changing the location of WiFi-2G nodes from $\mathrm{JN}$ to $\mathrm{GM}$ reduced the $C_{68} \quad 0.2 \mathrm{~dB}$. The $\mathrm{CI}_{68}$ remains constant $(4 \mathrm{~dB})$ for LTE 2600 (changing the location of the nodes from BT to DL). It can be concluded: 1) the designed DPDE is less affected by the wearer's body compared to single nodes on the body. 2) The optimized location of the nodes for the DPDE (based on calibration in the anechoic chamber) [8] can be used in diffuse fields as well.

Figure 6 depicts the mean on-body AA in diffuse fields for the optimized locations of the nodes for WiFi-2G and LTE 2600 (JN and BT). The designed DPDE has a mean AA (for the average over front and back) of $1.93 \mathrm{~cm}^{2}$ and $1.65 \mathrm{~cm}^{2}$, for WiFi-2G and LTE 2600, respectively. In a diffuse environment, a $P_{r}$ of $1 \mu \mathrm{W}$ on the antennas (nodes) corresponds to a mean $S_{i n c}$ of 5.2 and $6.1 \mathrm{~mW} . \mathrm{m}^{-2}$ for WiFi$2 \mathrm{G}$ and LTE 2600, respectively.
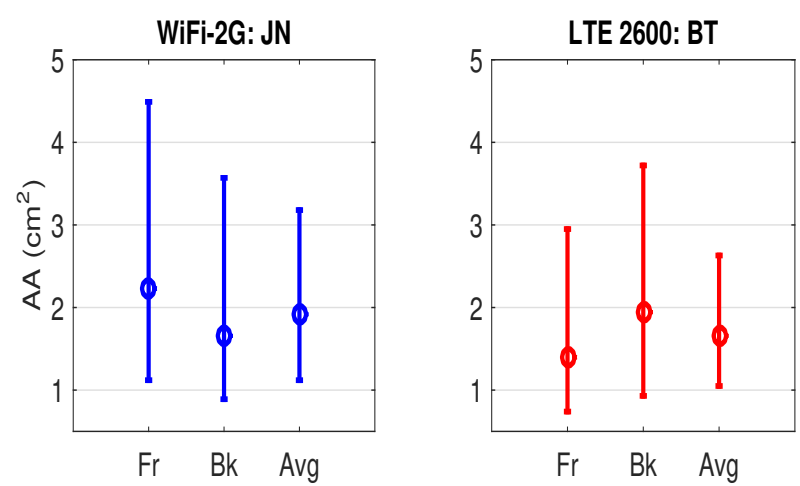

Figure 6. The logarithmic mean of on-body antenna aperture (AA) and 68\% confidence interval for the optimized location of the nodes on body. Fr:front; Bk:back; Avg:average over front and back.

\subsection{Validation in a real indoor environment}

Table 1 lists the measured $S_{i n c}$ for WiFi-2G in the office environment using DPDE and the spectrum analyzer. The AA values for calibration of the DPDE in the anechoic chamber $\left(A A_{A N}\right)$ [8] and in the RC $\left(A A_{R C}\right)$ are used to obtain the actual incident power density on-body. The actual $S_{\text {inc }}$ should be in between both estimates but its exact value cannot be calculated, since the contribution of specular and diffuse fields in real environments is not known. On the first location the measured $S_{i n c}$ is $-54.24 \mathrm{dBW} / \mathrm{m}^{2}$. The DPDE estimates that the actual $S_{i n c}$ on body is between $56.9 \mathrm{dBW} / \mathrm{m}^{2}$ (specular) and $-55.5 \mathrm{dBW} / \mathrm{m}^{2}$ (DMC). Similarly, for the measured values at the second location, the actual power density on-body is estimated between -55.7 to $-57.1 \mathrm{dBW} / \mathrm{m}^{2}$. The results show that the DPDE is working properly. The designed DPDE has an on-body detection limit of $3 \mu \mathrm{W} / \mathrm{m}^{2}$.

Table 1. Measurement of $S_{i n c}$ using the DPDE and the triaxial antenna and a spectrum analyzer (SA) in the WiFi-2G band. Loc:location of measurement; AN:anechoic chamber; RC:reverberation chamber; DeL:on-body detection limit.

\begin{tabular}{cccc}
\hline$S_{\text {inc }}\left(d B W / m^{2}\right)$ & $A A_{A N}$ & $A A_{R C}$ & SA \\
\hline Loc 1 & -56.9 & -55.49 & -54.24 \\
Loc 2 & -57.13 & -55.71 & -54.16 \\
\hline AA $\left(\mathrm{cm}^{2}\right)$ & 2.68 & 1.93 & - \\
DeL $\left(\mu W / m^{2}\right)$ & 1.87 & 2.59 & - \\
\hline
\end{tabular}

\section{Conclusions}

A dual band wearable personal distributed exposure meter (DPDE) is proposed to measure the human exposure in diffuse indoor environments. The DPDE consists of four nodes that are worn on body measuring the incident power density at WiFi-2G and LTE 2600 downlink bands. The location of the nodes of the DPDE is optimized based on calibrations in the anechoic chamber. Calibration of the DPDE is the RC confirms that the optimized configuration of the DPDE can be used for an improved assessment of personal diffuse exposure in indoor environments. The designed meter has a measurement uncertainty of maximum $4.5 \mathrm{~dB}$ on its antenna aperture. The WiFi-2G signals are measured by the DPDE in an indoor office building. The results are compared with the measurements of a tri-axial antenna and a spectrum analyzer. The results are in good agreement. The designed DPDE has an on-body detection limit of $3 \mu \mathrm{W} / \mathrm{m}^{2}$.

\section{Acknowledgements}

This research was funded by the Research Foundation Flanders (FWO) under grant agreement No G003415N and the French National Research Program for Environmental and Occupational Health of ANSES (2015/2 RF/07) as part of project ACCEDERA. A.T. has received funding from 
the European Union's Horizon 2020 research and innovation programme under the Marie Skłodowska-Curie grant agreement No 665501 with the FWO. A.T. is an FWO [PEGASUS $]^{2}$ Marie Skłodowska-Curie Fellow. S.A. is a Post-Doctoral Fellow of the FWO.

\section{References}

[1] C. Aliaga, "How is the time of women and men distributed in europe. statistics in focus, population and social conditions. issn 1024-4352, ks-nk-06-004-en-n, eurostat Luxembourg," 2006, http://www.unece.org/fileadmin/DAM/stats/gender/ timeuse/DataReports/How_is_the_time_of_Women_ and_Men.pdf, accessed on 30 January 2018.

[2] World Health Organization (WHO), "Radio frequency (RF) fields research agenda," http://www.who.int/ peh-emf/research/agenda/en/, accessed on 30 January 2018.

[3] J. Andersen, J. Nielsen, G. Pedersen, G. Bauch, and M. Herdin, "Room electromagnetics," Antennas and Propagation Magazine, IEEE, vol. 49, no. 2, pp. 2733, April 2007

[4] J. Poutanen, J. Salmi, K. Haneda, V. Kolmonen, and P. Vainikainen, "Angular and shadowing characteristics of dense multipath components in indoor radio channels," Antennas and Propagation, IEEE Transactions on, vol. 59, no. 1, pp. 245-253, Jan 2011.

[5] J. Blas, F. A. Lago, P. Fernández, R. M. Lorenzo, and E. J. Abril, "Potential exposure assessment errors associated with body-worn rf dosimeters," Bioelectromagnetics, vol. 28, no. 7, pp. 573-576, 2007.

[6] J. F. Bolte, "Lessons learnt on biases and uncertainties in personal exposure measurement surveys of radiofrequency electromagnetic fields with exposimeters," Environment International, vol. 94, no. Supplement C, pp. 724 - 735, 2016.

[7] A. Thielens, H. De Clercq, S. Agneessens, J. Lecoutere, L. Verloock, F. Declercq, G. Vermeeren, E. Tanghe, H. Rogier, R. Puers, L. Martens, and W. Joseph, "Personal distributed exposimeter for radio frequency exposure assessment in real environments," Bioelectromagnetics, vol. 34, no. 7, pp. 563-567, 2013.

[8] R. Aminzadeh, A. Thielens, S. Agneessens, P. Van Torre, M. Van den Bossche, S. Dongus, M. Eeftens, A. Huss, R. Vermeulen, R. de Seze, P. Mazet, E. Cardis, H. Rogier, M. Röösli, L. Martens, and W. Joseph, "A multi-band body-worn distributed radio-frequency exposure meter: Design, on-body calibration and study of body morphology," Sensors, vol. 18, no. 272, 2018. [Online]. Available: http://www.mdpi.com/1424-8220/18/1/272
[9] R. Aminzadeh, A. Thielens, A. Bamba, L. Kone, D. P. Gaillot, M. Lienard, L. Martens, and W. Joseph, "Onbody calibration and measurements using personal radiofrequency exposimeters in indoor diffuse and specular environments," Bioelectromagnetics, vol. 37, no. 5, pp. 298-309, 2016.

[10] S. Agneessens, "Coupled eighth-mode substrate integrated waveguide antenna: Small and wideband with high-body antenna isolation," IEEE Access, 2017.

[11] S. Aerts, D. Deschrijver, W. Joseph, L. Verloock, F. Goeminne, L. Martens, and T. Dhaene, "Exposure assessment of mobile phone base station radiation in an outdoor environment using sequential surrogate modeling," Bioelectromagnetics, vol. 34, no. 4, pp. 300-311, 2013. 